\title{
A metabolomics approach to characterize raw, pasteurized, and ultra-high temperature milk using ultra-performance liquid chromatography-quadrupole time-of-flight mass spectrometry and multivariate data analysis
}

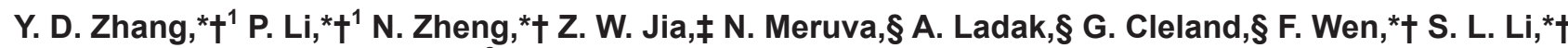 \\ S. G. Zhao, ${ }^{*} \dagger$ and J. Q. Wang ${ }^{*} \dagger^{2}$ \\ *Ministry of Agriculture Laboratory of Quality and Safety Risk Assessment for Dairy Products (Beijing), Institute of Animal Science, \\ Chinese Academy of Agricultural Sciences, Beijing 100193, China \\ †State Key Laboratory of Animal Nutrition, Institute of Animal Science, Chinese Academy of Agricultural Sciences, Beijing 100193, China \\ ¥Waters China, Shanghai 201206, China \\ $\S$ Waters Corporation, Milford, MA 01757
}

\begin{abstract}
We developed a metabolomics workflow using ultraperformance liquid chromatography coupled to quadrupole time-of-flight mass spectrometry to determine the effect of thermal treatment on milk composition and metabolites based on multivariate data analysis. We analyzed raw, pasteurized, and UHT milk samples. The samples were first centrifuged to remove the fat layer and mixed with methanol to precipitate proteins. Subsequently, the supernatant was analyzed by ultra-performance liquid chromatography coupled to quadrupole time-of-flight mass spectrometry in electrospray negative mode. Mass spectral data were acquired in $\mathrm{MS}^{\mathrm{E}}$ mode, a technique whereby both precursor and fragment mass spectral are simultaneously acquired by alternating between low and high collision energy (CE) during a single analytical run, to enable metabolite identification. Based on multivariate data analysis, these markers were significantly affected by thermal treatment. Among the 8 potential markers, we identified 7 oxylipids (9-hydroxydecanoic acid, 12-hydroxydodecanoic acid, 2-hydroxymyristic acid, 3-hydroxytetradecanoic acid, 5-hydroxyeicosatetraenoic acid, 3-hydroxyhexadecanoic acid, and 10-hydroxyoctadecanoic acid) and 1 phospholipid (LysoPE, hexadecanoyl-lysophosphatidylethanolamine). The oxylipids seemed to be adequate for distinguishing UHT milk from raw and pasteurized milk. The structures of the 8 potential markers were identified and characterized
\end{abstract}

Received January 14, 2018

Accepted April 27, 2018.

${ }^{1}$ These authors contributed equally to this article.

${ }^{2}$ Corresponding author: wangjiaqi@caas.cn using informatics software. Our metabolomics workflow provides a fast approach for the identification of various types of milk.

Key words: metabolomics, milk, pasteurized, ultra-high temperature, ultra-performance liquid chromatography-quadrupole time-of-flight mass spectrometry

\section{INTRODUCTION}

Milk and dairy products are major food commodities due to their nutritional value (Milkovska-Stamenova and Hoffmann, 2016). Milk's neutral $\mathrm{pH}$ value and high water activity make it a suitable growth medium for several microorganisms, including pathogens (Claeys et al., 2013). Raw milk is generally sterilized by thermal treatment (e.g., pasteurization and UHT treatment) to extend its shelf life and ensure safety. Pasteurization, which destroys harmful microorganisms, involves heat treatment at $185^{\circ} \mathrm{F}\left(85^{\circ} \mathrm{C}\right)$ for $15 \mathrm{~s}$; UHT treatment, which inactivates pathogenic bacteria and bacterial spores for long-term storage, involves heat treatment at $275^{\circ} \mathrm{F}\left(135^{\circ} \mathrm{C}\right)$ for $15 \mathrm{~s}$.

Several researchers have investigated the effect of thermal treatment on milk composition. Changes in protein (Van Boekel, 1998; Corredig and Dalgleish, 1999; Pizzano et al., 2012; Milkovska-Stamenova and Hoffmann, 2016), lactose (Van Boekel, 1998), and vitamins (Van Boekel, 1998; Cappozzo et al., 2015) have been reported. However, the effect of thermal treatment on the nutrient profile of milk remains unclear.

Milk metabolomics are affected by complex matrices (Jensen, 1995, 2002), animal species (e.g., cow, goat, and sheep), thermal treatment, cow status (Auldist et al., 1998; Dewhurst et al., 2003), and farming systems (Ellis et al., 2006). Several techniques (liquid chroma- 
tography, GC, MS, nuclear magnetic resonance, and infrared spectroscopy) have been used to evaluate the quality, safety, and nutritional value of milk (Nicholson et al., 1999). Mass spectometry-based "omics" approaches (e.g., metabolomics, lipidomics, nutrigenomics, and proteomics) allow the screening of food allergens, assessment of food quality, and identification of contaminants or toxins (Herrero et al., 2012). Liquid chromatography-MS and GC-MS generate comprehensive metabolomics profiles, particularly for small metabolites $(<1 \mathrm{kDa})$ in complex matrices. Quadrupole time-of-flight (QTOF) MS provides accurate mass data for the identification of biomarkers and the structural elucidation of unknown compounds.

In this study, we developed an ultra-performance liquid chromatography (UPLC) QTOF MS method to assess the effect of different thermal treatments on the composition and nutritional quality of milk using multivariate data analysis. Potential biomarkers were further identified using informatics software.

\section{MATERIALS AND METHODS}

\section{Sample Collection and Processing}

Ten different batches of raw milk were collected from a commercial dairy plant over $10 \mathrm{~d}$ to ensure representative sampling. The samples were stored in sterile glass bottles in dry ice until sample processing. Matched pasteurized and UHT milk samples were generated from the 10 batches of raw milk within $24 \mathrm{~h}$ after milking. Raw milk was cooled to $4^{\circ} \mathrm{C}$ and subsequently subjected to pasteurization at $85^{\circ} \mathrm{C}$ for $15 \mathrm{~s}$ (Shanghai Nanhua Transducer Manufacture Co. Ltd., Shanghai, China) or UHT at $135^{\circ} \mathrm{C}$ for $15 \mathrm{~s}$ (Flex-NG, Tetra Pak, Pully, Switzerland). Following pasteurization and UHT, the samples were stored at $-80^{\circ} \mathrm{C}$. Pooled samples were prepared by mixing matched individual milk samples in a 1:1:1 ratio.

\section{Sample Pretreatment}

Approximately $2 \mathrm{~mL}$ of thawed milk samples was added to $10-\mathrm{mL}$ centrifuge tubes and centrifuged at $4,000 \times g$ for $20 \mathrm{~min}$ at $4^{\circ} \mathrm{C}$ to remove the top fat layer, which contained high-molecular-weight compounds. The supernatant was collected and centrifuged to remove the fat layer, and the resulting supernatant (skim milk) was collected. The skim milk was mixed with 3 $\mathrm{mL}$ of precooled methanol and centrifuged at 14,000 $\times g$ for $15 \mathrm{~min}$ at $4^{\circ} \mathrm{C}$ to precipitate proteins. Finally, $5 \mu \mathrm{L}$ of supernatant devoid of protein was used for UPLC-QTOF MS analysis.

\section{UPLC-QTOF MS Analysis}

The UPLC was performed using a Waters Acquity UPLC I-Class (Waters Corp., Milford, MA) equipped with a $2.1 \times 100-\mathrm{mm}$ HSS T3 column of $1.8-\mu \mathrm{m}$ particle size (Waters Corp.). Gradient elution at $0.5 \mathrm{~mL} /$ min was performed using $0.1 \%$ formic acid in water (solvent A) and $0.1 \%$ formic acid in acetonitrile (solvent $\mathrm{B}$ ). Initial solvent composition was $1 \%$ solvent B followed by a linear gradient over 10 min to $95 \%$ solvent B. This $95 \%$ solvent ratio was held for $2 \mathrm{~min}$ followed by a second linear gradient to $99 \%$ solvent B in $1 \mathrm{~min}$. The column was allowed to return $(0.1 \mathrm{~min})$ to the initial gradient conditions (1\% solvent B) over 2 min. The temperatures of the column oven $\left(40^{\circ} \mathrm{C}\right)$ and autosampler tray $\left(10^{\circ} \mathrm{C}\right)$ were controlled. For analysis, $5 \mu \mathrm{L}$ of sample was injected onto the column.

The UPLC system was connected to a QTOF MS (Waters Xevo G2-XS, Waters Corp.) equipped with an electrospray interface. Full-scan data were collected from 50 to 1,200 Da, and the data were acquired under low collision energy (CE) of $4 \mathrm{eV}$ and high $\mathrm{CE}$ of 10 to $50 \mathrm{eV}$. The capillary voltage was $1 \mathrm{kV}$, and the cone voltage was $30 \mathrm{~V}$ with cone and desolvation gas flows set to 50 and $1,000 \mathrm{~L} / \mathrm{h}$, respectively. The source and desolvation temperatures were set to 150 and $550^{\circ} \mathrm{C}$, respectively. The MS was operated at data-independent acquisition $\mathrm{MS}^{\mathrm{E}}$ mode to acquire accurate mass precursor and product ion information. Each sample was analyzed independently using electrospray positive and negative mode to increase the metabolome coverage.

\section{Sample Analysis}

All samples were analyzed over the course of $4 \mathrm{~d}$ with 10 technical replicates for each milk group in electron spray ionization positive and negative modes. Within each batch, the samples were completely randomized to remove systematic error and minimize the effects of sample and analytical variability. The chromatographic column was conditioned with the pooled mixture before sample analysis. The pooled samples were analyzed before, during, and after batch analyses to ensure that the mass resolution, mass accuracy, and instrument response met the acceptance criteria throughout the analyses.

\section{Data Processing}

Spectrometric data acquired by Masslynx (Waters Corp., Milford, MA) were processed with Progenesis QI software (Waters Corp.) for biomarker discovery and multivariate data analysis. Progenesis QI provides 


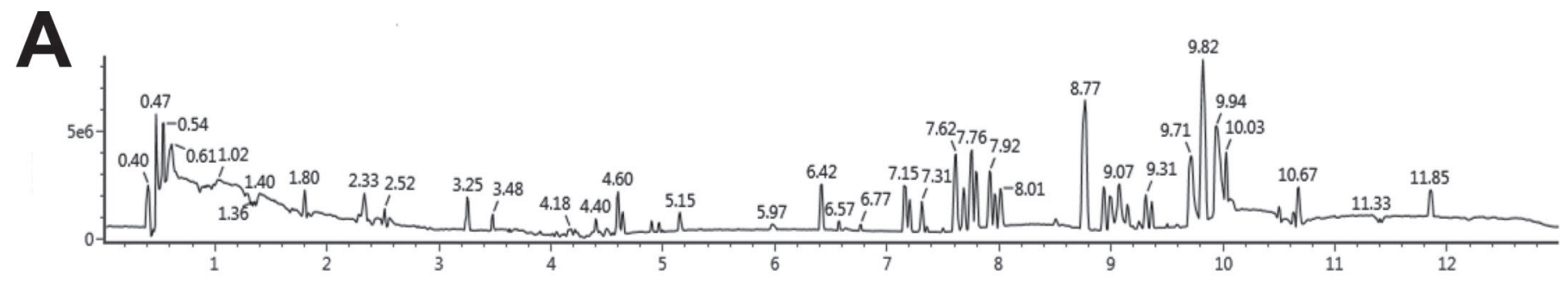

B

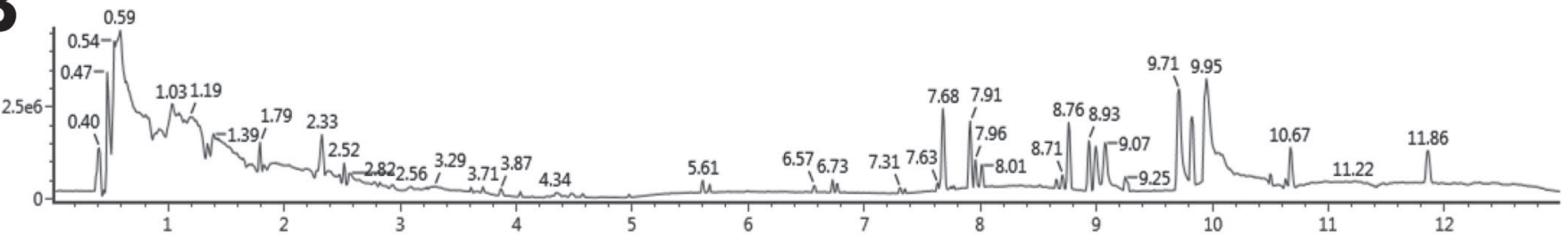

C

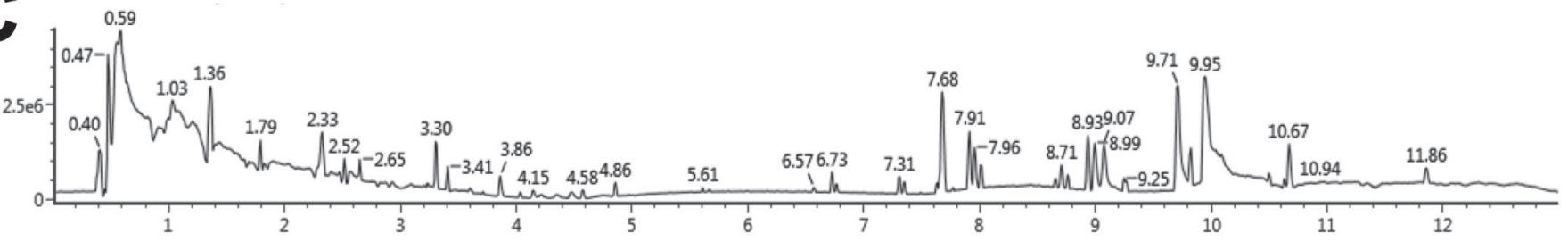

D

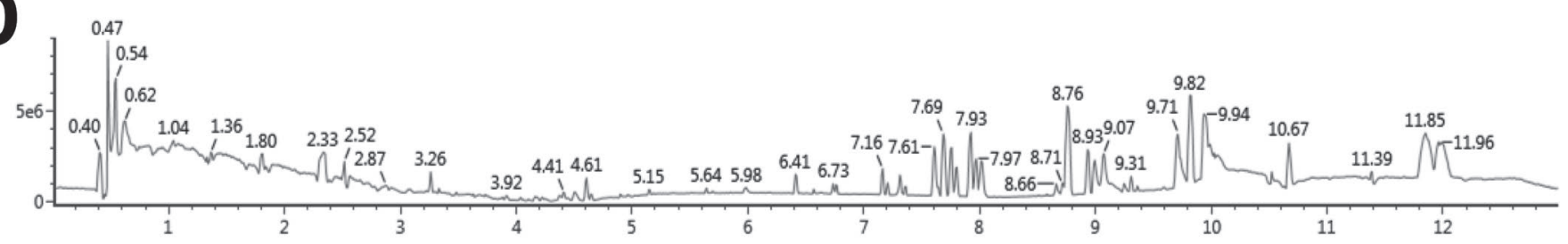

Retention time (min)

Figure 1. Typical base peak intensity chromatograms of raw (A), pasteurized (B), UHT (C), and pooled (D) milk samples from ultraperformance liquid chromatography-quadrupole time-of-flight MS in ESI- ionization mode.

an automatic workflow for spectral alignment, peak detection, and normalization of raw chromatography and mass spectral data for biomarker characterization. Principal components analysis (PCA), structural elucidation tools, and other statistical tools were implemented.

\section{RESULTS AND DISCUSSION}

\section{Analysis of Milk Metabolomics Profiling}

The 2 main challenges in milk metabolomics research are efficient separation and precise characterization of a wide range of compounds present in milk samples. Compared with HPLC, UPLC provides superior separation results in terms of resolution and number of peaks detected (Ortega et al., 2010; Yanamandra et al., 2010). We used a reversed-phase UPLC separation and a sub $2-\mu \mathrm{m}$ column to increase peak capacity, chromatographic resolution, and specificity for metabolic profiling. A typical base peak intensity chromatogram obtained in ESI- mode provides sharper peaks, which increases the chromatographic resolution and ability to detect more candidate markers and metabolites (Figure 1). Moreover, $>15,000$ ions detected from a pooled sample in ESI- mode represent the distribution of masses and retention times encountered for this sample.

Sharper peaks from UPLC separation increase the resolution and numbers of peaks and demand fast acquisition, making QTOF MS ideal for unbiased metabolic profiling. The $\mathrm{MS}^{\mathrm{E}}$ technique was used to acquire comprehensive mass spectral data, including accurate 
mass precursor and fragment ions, isotope distribution, and adducts by alternating between low and high CE during a single analytical run. The protonated molecular ion $[\mathrm{M}+\mathrm{H}]+$ information acquired from the low and high $\mathrm{CE}$ was used to analyze the metabolic profile and characterize the marker structures. The experimental approaches presented in this study may be significant for studying metabolites and eliminating the need to rerun samples to obtain more MS data.

\section{Multivariate Data Analysis of UPLC-QTOF MS Data}

We performed PCA and generated loading plots on the metabolite profiles obtained from the 3 milk samples. Progenesis QI software was used for preprocessing and multivariate statistical analysis. The spectral regions before $0.5 \mathrm{~min}$, which represented dead volume, and after $13 \mathrm{~min}$ of gradient elution were excluded from the data analysis. Following alignment, peak detection, and deconvolution, $>15,000$ compound ions were detected in ESI- mode. Figure 2 shows the PCA score plot clearly classifying the raw, pasteurized, and UHT milk samples. The pooled milk samples and centralized pooled composite sample indicate a statistically relevant data set. The data were further investigated by generating a loading plot (Figure 3 ) to identify metabolites with significant changes. A distinct clustering among the raw, pasteurized, and UHT milk samples was achieved, which suggests that the metabolites significantly changed after thermal treatment.

\section{Identification of Potential Biomarkers}

The application of $\mathrm{MS}^{\mathrm{E}}$ data with accurate mass for precursor and fragment ions, retention time, elemental composition, isotopic distribution, and match between theoretical fragmentation and measured fragmentation was used to improve confidence in metabolite identification. The initial metabolite or marker table consisted of $>15,000$ compound ions in electrospray negative mode. Compounds with significant statistical differences $(P<0.5$; variable importance in the projection $>1)$ and maximum intensity difference between one or more groups (max-fold change) were recorded. Prominent markers in each sample were identified using loading plots (Figure 3) generated using EZ Info software (Waters Corp.). The furthest markers from the origin contributed significantly to the clustering of different groups. Corresponding loading plots revealed the possible biomarkers with retention time and mass: charge ratio $(\mathrm{m} / \mathrm{z})$ pairs of 4.73 and $188.1412,5.84$ and 439.5237, 6.01 and 216.1725, 7.07 and 244.2038, 7.19 and $244.2038,8.03$ and $320.2351,8.31$ and 272.2338 , and 9.38 and 300.4766 .

We used Progenesis QI software to identify potential biomarkers based on in-house and public databases. With the search engine Progenesis Metascope, structure information was obtained by searching HMDB (http://www.hmdb.ca/), Chemspider (http://www .chemspider.com/), and KEGG (http://www.kegg $. \mathrm{com} /)$ databases. If the selected database contained structures and theoretical fragmentation of the mole-

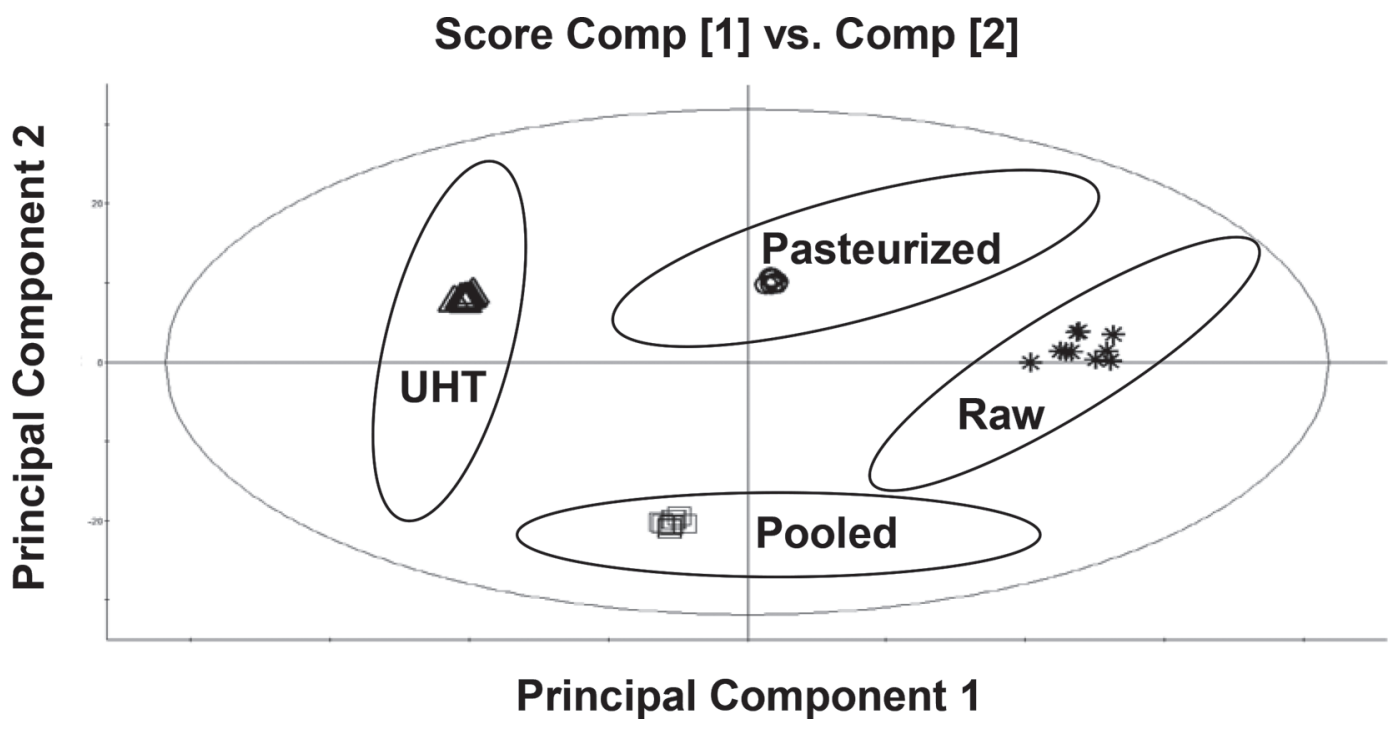

Figure 2. Principal component (PC) analysis plot of 40 milk samples representing 10 replicates from each of the 4 groups (raw, pasteurized, UHT, and pooled milk samples). The plot is based on the first 2 PC for data acquired in ESI- mode. There were significant changes in metabolomics after thermal treatment. 


\section{Loading Comp [1] vs. Comp [2]}

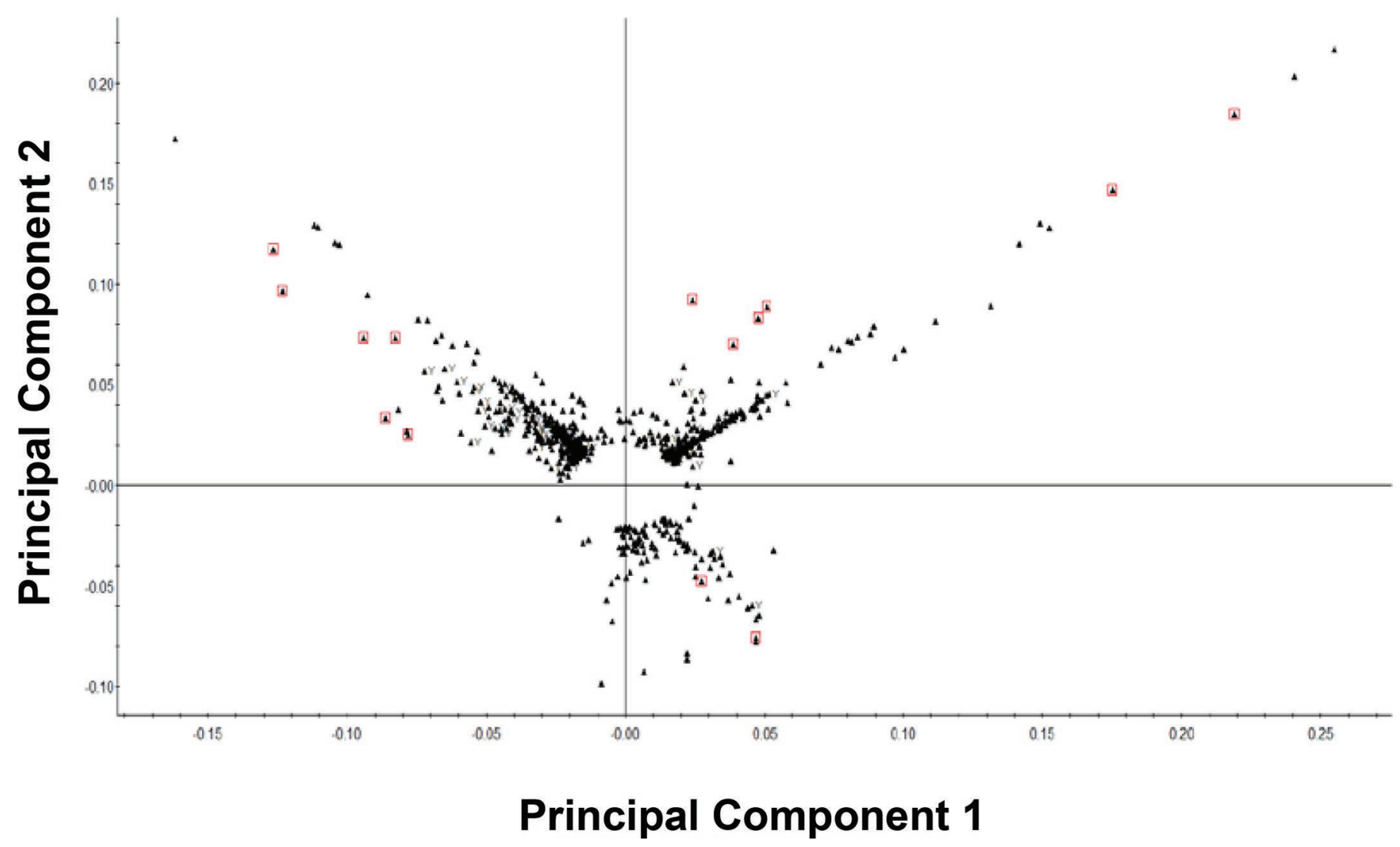

Figure 3. Loading plots of milk samples representing 10 replicates from each of the 3 groups (raw, pasteurized, and UHT milk samples). The variables marked with squares $(\square)$ are the metabolites selected as potential biomarkers. Color version available online.

cule, a fragmentation score was used to rank potential identifications of high-energy accurate mass fragment ions to the theoretical dissociation of the molecule. A list of potential identifications was automatically generated and scored using mass accuracy, isotope distribution, retention time, and fragment matching. A total of 92 metabolites were identified in the 3 milk samples based on accurate elemental composition and available databases. After manual comparison based on Figure 3, the identities of 8 potential biomarkers were revealed (Table 1). A total of 7 oxylipids (9-hydroxydecanoic acid, 12-hydroxydodecanoic acid, 2-hydroxymyristic acid, 3-hydroxytetradecanoic acid, 5-hydroxyeicosatetraenoic acid, 3-hydroxyhexadecanoic acid, and 10-hydroxyoctadecanoic acid) appeared to be adequate markers to distinguish UHT milk from raw and pasteurized milk. Oxylipids are potent bioactive lipid mediators with essential roles in normal physiology and function. Oxylipids formed from PUFA are important mediators of the effects of PUFA on the body (Pickens et al., 2017). Oxylipids are endogenously synthesized by cytochrome P450 enzymes (Arnold et al., 2010), cyclooxygenase (Smith et al., 2000), lipoxygenase (Mabalirajan et al., 2012), and nonenzymatic pathways such as reactive oxygen species, especially during oxidative stress. For example, 5-hydroxyeicosatetraenoic acid is the product of 5-lipoxygenase (Mavangira et al., 2015). Figure 4 shows the resulting spectrum for 5-hydroxyeicosatetraenoic acid with its elemental composition, precursor ion, and associated product ions.

\section{Positive ESI Data}

The same methodology was applied for the positive ionization data (i.e., PCA and loading plots to obtain potential markers). Even though separation was achieved in the PCA model, the relevance of markers was comparatively slightly lower. Therefore, data from ESI+ were discarded. 
Table 1. Prominent markers identified in electron spray ionization negative data of raw, pasteurized, and UHT milk samples

\begin{tabular}{|c|c|c|c|c|c|c|}
\hline Compound & $\begin{array}{l}\text { Retention } \\
\text { time (min) }\end{array}$ & Formula & $\begin{array}{l}\text { Accurate } \\
\text { mass (Da) }\end{array}$ & $\begin{array}{l}\text { Product } \\
\text { ion } 1\end{array}$ & $\begin{array}{l}\text { Product } \\
\text { ion } 2\end{array}$ & Occurrence \\
\hline 9-Hydroxydecanoic acid & 4.73 & $\mathrm{C}_{10} \mathrm{H}_{20} \mathrm{O}_{3}$ & 188.1412 & 144.1514 & 116.1201 & UHT milk \\
\hline LysoPE, hexadecanoyl-lysophosphatidylethanolamine & 5.84 & $\mathrm{C}_{20} \mathrm{H}_{42} \mathrm{NO}_{7} \mathrm{P}$ & 439.5237 & 156.1878 & 100.1252 & $\begin{array}{l}\text { Pasteurized } \\
\text { and UHT milk }\end{array}$ \\
\hline 12-Hydroxydodecanoic acid & 6.01 & $\mathrm{C}_{12} \mathrm{H}_{24} \mathrm{O}_{3}$ & 216.1725 & 172.1827 & 144.1514 & UHT milk \\
\hline 2-Hydroxymyristic acid & 7.07 & $\mathrm{C}_{14} \mathrm{H}_{28} \mathrm{O}_{3}$ & 244.2038 & 200.2140 & 116.1201 & UHT milk \\
\hline 3-Hydroxytetradecanoic acid & 7.19 & $\mathrm{C}_{14} \mathrm{H}_{28} \mathrm{O}_{3}$ & 244.2038 & 228.2089 & 200.2140 & UHT milk \\
\hline 10-Hydroxyoctadecanoic acid & 9.38 & $\mathrm{C}_{18} \mathrm{H}_{36} \mathrm{O}_{3}$ & 300.4766 & 256.2766 & 214.2297 & UHT milk \\
\hline
\end{tabular}

\section{CONCLUSIONS}

We developed a high-throughput method to screen profiles for both untargeted and targeted metabolomics in milk samples by integrating unbiased $\mathrm{MS}^{\mathrm{E}}$ data acquisition based on UPLC-QTOF MS and multivariate data analysis. Inspired by the rich qualitative and quantitative data, we quickly obtained the changes in metabolomics profiles in 3 milk samples, identified 92 metabolites, and characterized 8 potential biomarkers in ESI mode. Based on multivariate data analysis, the markers significantly changed in the milk samples after thermal treatment. Finally, we identified 7 oxylipids that distinguish UHT milk from raw and pasteurized milk: 9-hydroxydecanoic acid, 12-hydroxydodecanoic acid, 2-hydroxymyristic acid, 3-hydroxytetradecanoic acid, 5-hydroxyeicosatetraenoic acid, 3-hydroxyhexadecanoic acid, and 10-hydroxyoctadecanoic acid. Our method provides a fast approach for the identification and elucidation of various milk metabolites.
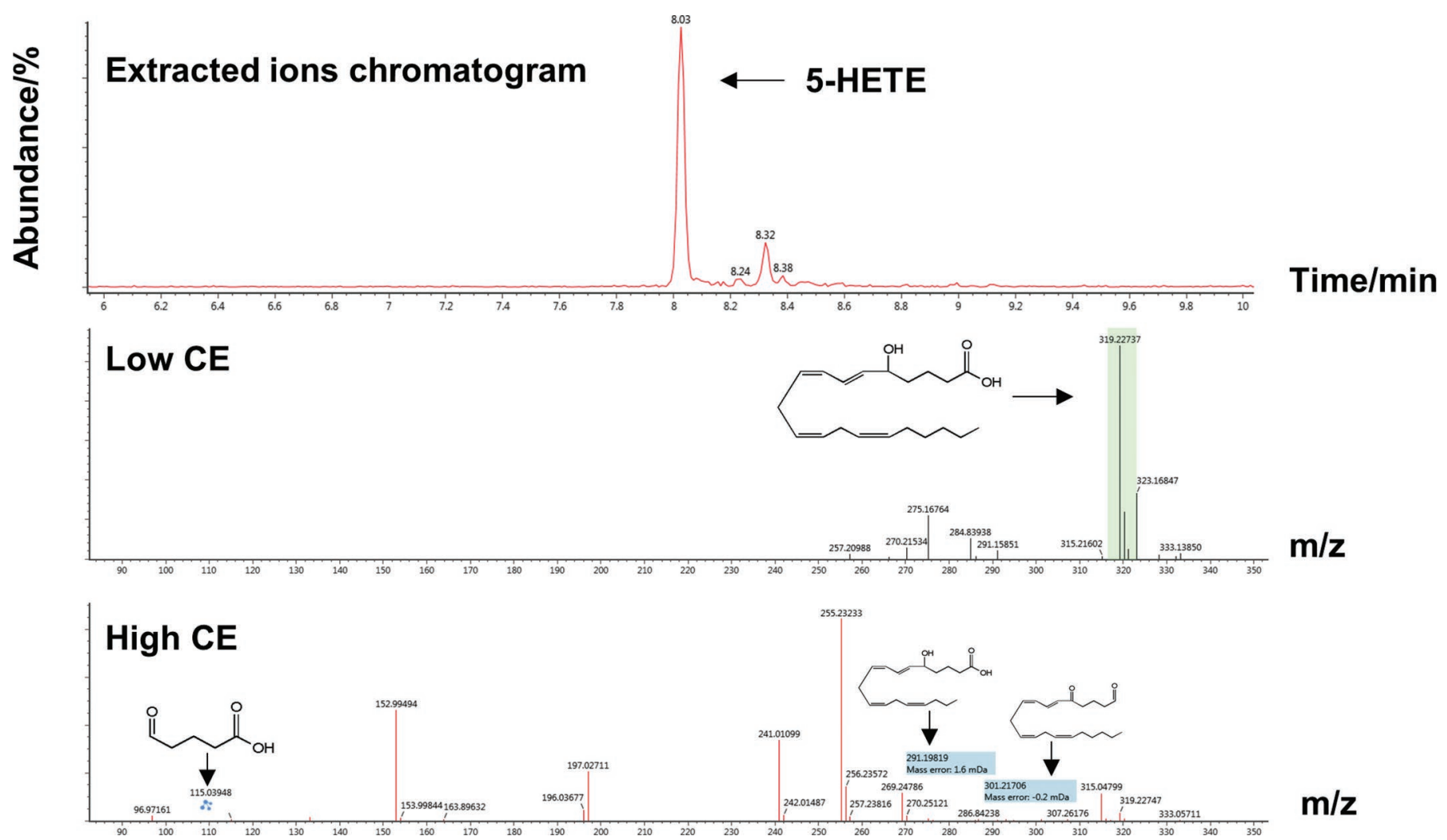

Figure 4. The extracted ions chromatogram of biomarker 5-hydroxyeicosatetraenoic acid (5-HETE) at 5.80 min showed elemental composition, precursor ion, and associated product ions in ESI- mode. Fragment ions of 5-HETE at mass:charge ratio $(\mathrm{m} / z) 319.2273$ are $m / z 171.1378$, 141.1280, and 101.0603. CE = collision energy. Color version available online. 


\section{ACKNOWLEDGMENTS}

This work was financially supported by the Special Fund for Agro-scientific Research in the Public Interest (201403071), Introduction of International Advanced Agricultural Science and Technology Program (2016X37), the Modern Agro-Industry Technology Research System of China (CARS-37), and the Agricultural Science and Technology Innovation Program (ASTIPIAS12), China.

\section{REFERENCES}

Arnold, C., M. Markovic, K. Blossey, G. Wallukat, R. Fischer, R. Dechend, A. Konkel, C. V. Schacky, F. C. Luft, D. N. Muller, M. Rothe, and W. H. Schunck. 2010. Arachidonic acid-metabolizing cytochrome P450 enzymes are targets of \{omega\}-3 fatty acids. J. Biol. Chem. 285:32720-32733.

Auldist, M. J., B. J. Walsh, and N. A. Thomson. 1998. Seasonal and lactational influences on bovine milk composition in New Zealand. J. Dairy Res. 65:401-411. https://doi.org/10.1017/ S0022029998002970.

Cappozzo, J. C., T. Koutchma, and G. Barnes. 2015. Chemical characterization of milk after treatment with thermal (HTST and UHT) and nonthermal (turbulent flow ultraviolet) processing technologies. J. Dairy Sci. 98:5068-5079.

Claeys, W. L., S. Cardoen, G. Daube, J. De Block, K. Dewettinck, K. Dierick, L. De Zutter, A. Huyghebaert, H. Imberechts, P. Thiange, Y. Vandenplas, and L. Herman. 2013. Raw or heated cow milk consumption: Review of risks and benefits. Food Control 31:251-262.

Corredig, M., and D. G. Dalgleish. 1999. The mechanisms of the heatinduced interaction of whey proteins with casein micelles in milk. Int. Dairy J. 9:233-236.

Dewhurst, R. J., W. J. Fisher, J. K. Tweed, and R. J. Wilkins. 2003. Comparison of grass and legume silages for milk production. 1. Production responses with different levels of concentrate. J. Dairy Sci. 86:2598-2611.

Ellis, K. A., G. Innocent, D. Grove-White, and M. Mihm. 2006. Comparing the fatty acid composition of organic and conventional milk. J. Dairy Sci. 89:1938-1950.
Herrero, M., C. Simó, V. García-Cañas, E. Ibáñez, and A. Cifuentes. 2012. Foodomics: MS-based strategies in modern food science and nutrition. Mass Spectrom. Rev. 31:49-69.

Jensen, R. G. 1995. Handbook of Milk Composition. Academic Press, San Diego, CA.

Jensen, R. G. 2002. The composition of bovine milk lipids: January 1995 to December 2000. J. Dairy Sci. 85:295-350.

Mabalirajan, U., A. Agrawal, and B. Ghosh. 2012. 15-Lipoxygenase eicosanoids are the putative ligands for vanilloid receptors and peroxisome proliferator-activated receptors (PPARs). Proc. Natl. Acad. Sci. USA 109:E1.

Mavangira, V., J. C. Gandy, C. Zhang, V. E. Ryman, A. D. Jones, and L. M. Sordillo. 2015. Polyunsaturated fatty acids influence differential biosynthesis of oxylipids and other lipid mediators during bovine coliform mastitis. J. Dairy Sci. 98:6202-6215.

Milkovska-Stamenova, S., and R. Hoffmann. 2016. Identification and quantification of bovine protein lactosylation sites in different milk products. J. Proteomics 134:112-126.

Nicholson, J. K., J. C. Lindon, and E. Holmes. 1999. "Metabolomics": Understanding the metabolic responses of living systems to pathophysiological stimuli via multivariate statistical analysis of biological NMR spectroscopic data. Xenobiotica 29:1181-1189.

Ortega, N., M. P. Romero, A. Macia, J. Reguant, N. Angle, J. R. Morello, and M. J. Motilva. 2010. Comparative study of UPLC-MS/ MS and HPLC-MS/MS to determine procyanidins and alkaloids in cocoa samples. J. Food Compos. Anal. 23:298-305.

Pickens, C. A., L. M. Sordillo, C. Zhang, and J. I. Fenton. 2017. Obesity is positively associated with arachidonic acid-derived 5- and 11-hydroxyeicosatetraenoic acid (HETE). Metabolism 70:177-191.

Pizzano, R., C. Manzo, M. A. Nicolai, and F. Addeo. 2012. Occurrence of major whey proteins in the $\mathrm{pH} 4.6$ insoluble protein fraction from UHT-treated milk. J. Agric. Food Chem. 60:8044-8050.

Smith, W. L., D. L. DeWitt, and R. M. Garavito. 2000. Cyclooxygenases: Structural, cellular, and molecular biology. Annu. Rev. Biochem. 69:145-182.

Van Boekel, M. A. J. S. 1998. Effect of heating on Maillard reactions in milk. Food Chem. 62:403-414.

Yanamandra, R., A. Chaudhary, S. R. Bandaru, B. Patro, Y. L. N. Murthy, P. A. Ramaiah, and C. S. P. Sastry. 2010. UPLC method for simultaneous separation and estimation of secnidazole, fluconazole and azithromycin in pharmaceutical dosage forms. J. Chem. 7:S363-S371. 